\title{
A Design of Automatic Equipment for Cigarette Sorting and Stacking
}

\author{
Liang Han a, Xianglei Dang b \\ School of Mechanical Engineering, Southeast University, Jiangning \\ District, Nanjing, 211189, China \\ aemail:melhan@seu.edu.cn, bemail: dangxianglei@seu.edu.cn
}

\begin{abstract}
Automatic equipment for cigarette sorting and stacking is applied to a recycling process of tobacco in cigarette factory. It takes the place of manual work, such as sorting and stacking cigarettes to transfer boxes. The equipment realizes the functions such as orientation, sorting, steering, and stacking of the cigarettes in proper order. Omron CP1H type PLC is selected as the control center of the equipment. The paper completes the design of mechanical structures and control system of the equipment, which achieves sorting and stacking automation and solves the problems of large labor input and low sorting efficiency.

Keywords: Cigarette Sorting; Cigarette Stacking; Tobacco Recycling; PLC

\section{Introduction}

In industrial production of cigarettes, a large number of cigarettes are inevitably rejected as defective products throughout the production processes. According to statistics, while a factory produces a box of cigarettes, it generates an average of $2.5 \mathrm{~kg}$ defective cigarettes. In other words, if the annual output is 45,000 boxes, it nearly generates 1125 tons of defective cigarettes per year. These cigarettes are automatically rejected by cigarette packing machines due to bending, lack of filter, duplicate rolls and short defects. Such a large number of defective cigarettes are ought to be recycled [1]. In order to maximize the degree of retaining the integrity of tobacco in the separation process, the equipment which applies a high-pressure air to blow tobacco out is mainly used in recycling process in cigarette factory. The input cigarettes are required to be stacked neatly without bending or breaking, and it only works under the condition that cigarette filters are palletized towards consensus direction.
\end{abstract}




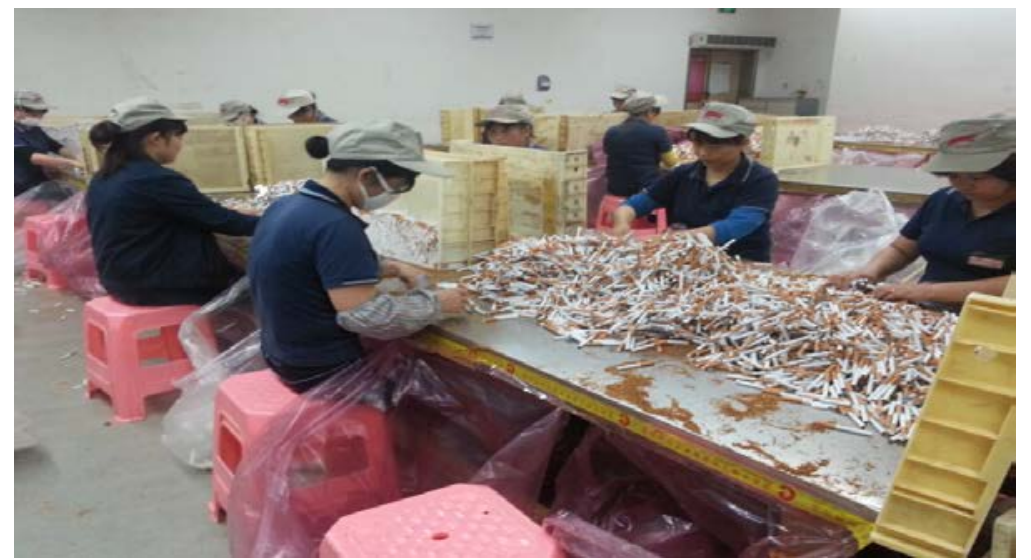

Fig.1. Workshop scene

According to the survey, there is no specific equipment to complete the work of above cigarettes sorting and stacking, and these work are done manually. Firstly, it needs to arrange a mess of cigarettes in a sequence and to reject seriously damaged cigarettes, and then to stack cigarettes into the same direction in transfer boxes. To complete the above sorting and stacking work, it requires a lot of labors. As is known, the work is time-consuming, inefficient and tedious, and workshop scene in a factory is shown in Fig.1.

Therefore, it is essential and urgent to design an equipment to sort and stack cigarettes automatically. In order to overcome the shortcomings of manual operation, the paper designs a highly automatic equipment for cigarette sorting and stacking.

\section{The Overall Design and Realization}

Automatic equipment for cigarette sorting and stacking mainly consists of cigarette feeding device, shaping device, steering device and stacking device. These parts are linked and integrated with Omron PLC as the control center. 


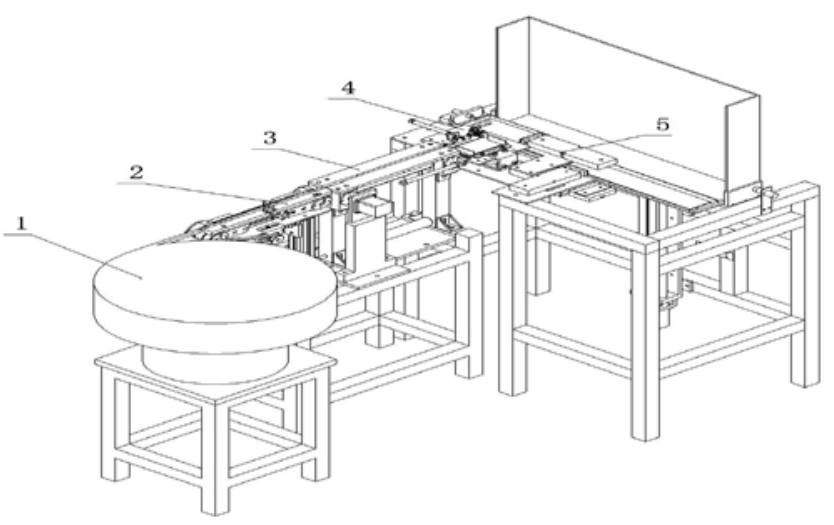

1.Vibratory bowl feeder; 2. First transmission section; 3 . Second transmission section; 4. Steering device; 5 . Stacking device

Fig.2. Overall design scheme

The vibratory bowl feeder is used as body of the feeding device, and the bowl is provided with a pretreated part for large bending cigarettes. The cigarettes are outputted in the longitudinal direction from the discharge port, and large bending cigarettes are not fed from the bowl. Optical fiber sensors and air nozzle are installed in the arc chute of the bowl [2-5], as is shown in Fig.3. Cigarettes move along the outer wall of the chute due to centrifugal force caused by vibration [6]. At this moment horizontal and vertical curvature of cigarettes are detected by two sensors. Substandard cigarettes are blown off by the air nozzle in order to prevent the bending cigarettes from blocking the exit of vibrating feeder, and then qualified cigarettes walk along the length of cigarette into first transmission section. While adjusting the distance between the feeder outlet and the first belt, cigarettes with short length can not enter the first transmission section.

The shaping device consists of first transmission section and second transmission section, and sorting part of bending cigarettes is designed on the first transmission section, as shown in Fig.4. The inclination degree of first transmission belt is adjusted to ensure the cigarettes to walk along the side of the baffle. The first and second optical fiber sensors are responsible for detecting cigarettes whether the branch is bent with the reflective amount of light received by optical fiber sensors. If the cigarette is bent upward, the first optical fiber sensor has a signal. If the cigarette is bent horizontally, the second optical fiber sensor has a signal. No matter any one of the two optical fiber sensors has a signal, air nozzle blows bending cigarettes off, and it is qualified for cigarettes to go into second transmission section. 

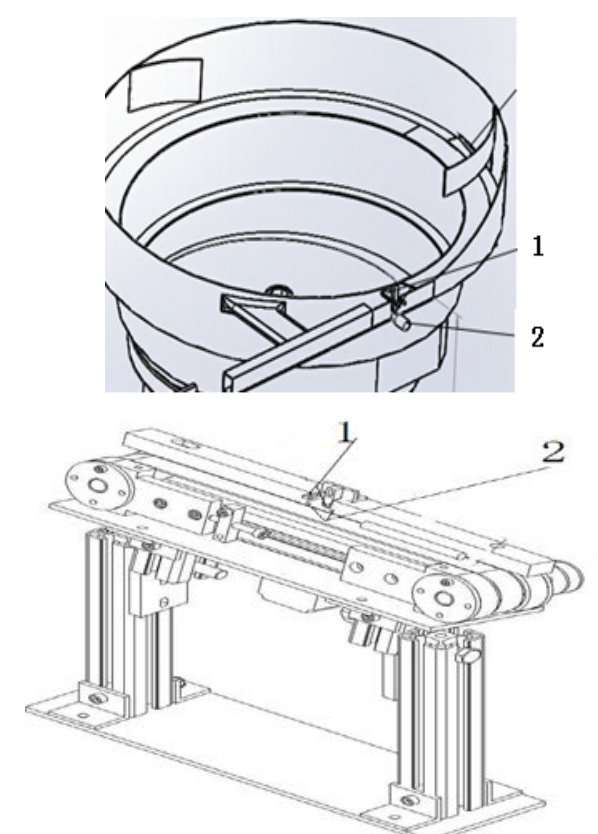

1. optical fiber sensors; 2 . air nozzle

Fig.3 Pretreatment of Bending Cigarettes

Fig.4 Secondary Treatment of

Bending Cigarettes

The steering device completes the steering function. One sensor detects whether cigarette moves in place, and another sensor detects the direction of cigarette filters. When the detection is finished, the cigarette is pushed into the chute by the plate driven by cylinder. The cigarette falls from the chute to rotating frame, and then it is decided whether to rotate or not on the basis of test results, and finally the cylinder pushes cigarette into the stacking device [7-10].

The stacking device receives cigarettes from the channel of steering device. After a cigarette is in place, it is pushed into the bottom surface of the platform, and then the cylinder pushes the cigarette under the cover. The distance between the bottom surface of the platform and the cover is nearly a diameter slightly larger than a cigarette. The width of cover is slightly less than the width of a transfer box. When the layer of cigarettes are pushed entirely below the cover, the second cylinder will push the entire layer of cigarettes into transfer box, and then the screw turns to reduce the height of a diameter of the cigarette.

The above work processes are completed automatically, workers only need to monitor equipment and replace transfer boxes. The sensor is fixed on the side of the transfer box frame. When transfer box falls below the bottom of the platform, the sensor is triggered to alert workers to replace the transfer box with sound and light alarms.

\section{Advantages and Innovation}


It is really effective to realize the bending detection of cigarettes, steering and stacking and other steps with fully automatic equipment based on the principles of modularization structure and automatic detection and control. Compared with the prior art, it has the following advantages:

1. High sorting efficiency. Even though skilled workers do these sorting and stacking operations, it is still difficult and unable to meet requirements of production schedule. Recycling efficiency is greatly improved with mechatronics method.

2. Lower costs. The structure is compact, and horizontal dimension of the entire device is less than 1.5 meters. In the realization of cigarette sorting and stacking, workers just need to do auxiliary work, which greatly reduces labor inputs and costs of production.

3. Stable and strong operability. The control system is made up of PLC and HMI with using a dedicated control panel. It is simple and convenient to operate, display and control.

4. Simple rotating route of cigarettes and low losses. The moving route of the cigarettes is simple. No secondary damage of tobacco is brought about. Compared with the traditional way which depends on workers to pick the cigarettes, it improves intact rate of recycling tobacco.

\section{Conclusion}

This paper proposes a new production concept about the pretreatment of tobacco recycling. It has lots of advantages such as simple principle, good applicability and strong operability. The machinery and control system improve the recycling efficiency of tobacco in cigarette factories. Moreover, it also solves the problem of tobacco damages which exists in the traditional production pattern, and brings notable economic benefits.

\section{Acknowledgement}

This work is supported by NSFC, project No.: 51275087.

\section{Reference}

[1] Lili Liu. Design of a New Type Processor for Incomplete Cigarette [J]. Light Industry Machinery, 2003,02: 73-75.

[2] Weimin Xu. Application of Photoelectric Technique in Detection Section of Tobacco Machinery [J]. Tobacco Science \& Technology, 2002(4)

[3] Yingchun Liu. Principles, Design \& Applications of Sensing Device.National University of Defence Technology Press, 1997.

[4] Faming Wang. Design of cigarette monitor system based On photoelectric [J].Instrumentation Customer.2008(4): 43-44.

[5] BLOSS Richard. JTM use Motoman to Stack Pails of Lubricants [J]. Industrial Robot,2006,33(1):24-26. 
[6] Jiachun Zhou. Operating Characteristic Analysis on Electromagnetic Vibration Hopper [M]. Equipment For Electronic Products Manufacturing, 1998,(3):8-10.

[7] Jiangping Mei. Technology and Applications on High-speed packaging robot [J]. Robot Technique and Application, 2007(5):18-20.

[8] MAHALIK Nitaigour P. Processing and Pack aging Automation Systems: a Review [J]. Sensing and Instrumentation for F ood Quality and Safety, 2009, $3(1): 12-25$.

[9] Hui Chen. Analysis of Automatic Packing and Palletizing Robot [J]. Science \& Technology Association Forum, 2009(10): 78-79.

[10] BLOSS Richard. Palletizing Candy Orders and Never Squeezing the Chocolates [J]. Assembly Automation, 2010,30(1):32- 35. 